\title{
Atom galleries for whispering atoms: binding atoms in stable orbits around an optical resonator
}

\author{
H. Mabuchi and H. J. Kimble \\ Norman Bridge Laboratory of Physics 12-33, California Institute of Technology, Pasadena, California 91125
}

Received December 12, 1993

\begin{abstract}
The external fields of optical whispering gallery modes may be used to confine atoms in stable orbits around a dielectric microsphere. As an example, a toroidal dipole-force trap (atom gallery) for three-level atoms is investigated, and the possibility of achieving an atomic (matter-wave) resonator is discussed. The extremely small electromagnetic mode volumes and high $Q$ 's of the whispering gallery modes should permit a circulating photon to be repeatedly absorbed and reemitted by a trapped whispering atom.
\end{abstract}

Several research groups, including our own, have demonstrated optical modes in quartz microspheres with quality factors $Q \approx 2 \times 10^{9} .1,2$ In these whispering gallery modes (WGM's) light circulates in a thin annular region near the equator, just inside the surface of the sphere. As in total internal reflection, WGM's have a small evanescent component that propagates just outside the surface of the sphere and hence provides access for interactions of the WGM with atoms in the external world. The high $Q$ 's and extremely small electromagnetic mode volumes that are simultaneously obtainable in quartz microspheres thus make them prime candidates for experiments in cavity quantum electrodynamics and quantum nondemolition detection. ${ }^{3,4}$ Indeed, microspheres of various compositions were the subject of many studies in nonlinear optics ${ }^{5,6}$ and optical levitation. ${ }^{7}$

Light circulating in a WGM with wavelength near an atomic resonance can exert strong forces on atoms in the evanescent region. In this Letter we describe a scheme for confining atoms in stable orbits by balancing the dipole forces associated with a pair of oppositely detuned WGM's. ${ }^{8}$ An interesting feature of such an atom-gallery trap is that atoms are confined to regions in which they can be strongly coupled to optical modes of the sphere. The coupling coefficient $g$ (corresponding to half the singlephoton Rabi frequency) for atoms interacting with WGM's can be made much larger than the atom's spontaneous emission rate $\gamma$ to noncavity modes. Furthermore the WGM's considered here can have sufficiently high $Q$ values that $g$ is likewise large compared with the damping rate $\kappa$ of the cavity field. In more physical terms, an initially excited atom can emit a photon that circulates in the WGM and is then reabsorbed, with this cycle repeating many times before decay by means of cavity leakage or spontaneous emission into free space. ${ }^{9}$ Note that the term whispering gallery mode originates in the analogy between optical modes in dielectric resonators and acoustic modes in large auditoriums. Hence the picture of the oscillatory exchange be- tween atom and WGM prompts us to speak of a whispering atom.

The perspective for cavity quantum electrodynamics with trapped atoms becomes even more exciting when one considers the possibility of using atoms with $\Delta p \leq \hbar / 2 \pi a$, where $a$ is the radius of atomic orbits. Such a system should exhibit resonance phenomena (as well as possible collective quantum effects) associated with the requirement that the de Broglie wavelength of atoms divide integrally into the trap circumference..$^{10,11}$ Note that the simultaneous quantization of atomic center-of-mass motion in conjunction with that of the internal atomic degrees of freedom and the cavity field presents a qualitatively new conceptual frontier.

Turning first to the stable confinement of atoms, we remark that the various forces and level shifts associated with externally applied fields ${ }^{12}$ and electromagnetic surface effects ${ }^{13}$ should lead to a number of ways in which to bind atoms in orbit around a dielectric sphere. To facilitate the discussion of possible trap configurations, let us establish a cylindrical coordinate system with axial coordinate $z$, radius $\rho$, and azimuthal angle $\phi$. The $z$ axis connects the poles of the microsphere, whereas atoms orbit just outside the surface of the sphere, principally in the equatorial plane $(z=0)$. We shall also refer to a spherical polar coordinate system with the same orientation ( $\theta=\pi / 2$ at the equator). In the following we drop the $\phi$ coordinate by assuming rotational invariance about the $z$ axis and consider the reduced problem of two-dimensional confinement in the $(\rho, z)$ plane.

The primary requirements for obtaining stable orbits are a long-range force that attracts atoms toward the sphere and a short-range repulsive force to prevent collisions with the surface. The forces must have functional forms that admit the formation of a minimum in the sum of their two-dimensional potentials. From the variety of possible schemes we discuss here the use of dipole forces ${ }^{14}$ associated with the simultaneous excitation of two different WGM's. Such a scheme can meet the above criteria if one chooses WGM's with wavelengths appropriate to the 
level structure of the atom of interest.

For example, for a three-level atom in a V configuration one of the WGM's may be red detuned with respect to the lower-energy atomic transition, whereas the other is blue detuned with respect to the higher-energy atomic transition, as depicted in Fig. 1. We suppose that only the $|0\rangle \leftrightarrow|1\rangle$ and $|0\rangle \leftrightarrow|2\rangle$ transitions are dipole allowed, with $\left(\omega_{1}, \omega_{2}\right)$ and $\left(\gamma_{1}, \gamma_{2}\right)$ as the corresponding transition frequencies and decay rates. Let the light fields be denoted as $\mathbf{E}_{a}(\mathbf{r}) \cos \left(\omega_{a} t\right)$ and $\mathbf{E}_{b}(\mathbf{r}) \cos \left(\omega_{b} t\right)$. Note that $\omega_{b}>\omega_{2}>\omega_{1}>\omega_{a}$. For simplicity we consider the case in which the detunings $\delta_{1,2} \equiv \omega_{a, b}-\omega_{1,2}$ satisfy $\left|\delta_{1}\right|=\left|\delta_{2}\right|=\delta$, and $\delta \ll \omega_{2}-\omega_{1}$. In the electric-dipole and rotating-wave approximations the position-dependent strength of the atom's interaction with the light fields is characterized by the two Rabi frequencies $\Omega_{1,2}(\mathbf{r})=\mathbf{d}_{1,2} \cdot \mathbf{E}_{a, b}(\mathbf{r}) / \hbar$, where $\mathbf{d}_{1,2}$ are the respective dipole transition matrix elements. One can then derive light shifts to first order in $\Omega_{1,2}(\mathbf{r}) / \delta$ by employing the dressed-state formalism ${ }^{14}$ and assuming $\delta \gg \Omega_{1,2}(\mathbf{r}) \gg \gamma_{1,2}$. The simple results are indicated in Fig. 1 ; the ground state $|0\rangle$ shifts by $\hbar\left(\Omega_{2}^{2}(\mathbf{r})-\Omega_{1}^{2}(\mathbf{r})\right) / 4 \delta$, level $|1\rangle$ shifts up by $\hbar \Omega_{1}^{2}(\mathbf{r}) / 4 \delta$, and level $|2\rangle$ shifts down by $-\hbar \Omega_{2}^{2}(\mathbf{r}) / 4 \delta$. In the limit considered we may assume that there is negligible excitation of atomic population and hence may identify the position-dependent ground-state light shift with the overall dipole potential $U_{d}(\mathbf{r})$.

The form of $U_{d}(\mathbf{r})$ thus follows immediately from the mode functions of the WGM's. The electric-field distribution inside the sphere for traveling-wave modes with TM polarization may be expressed in spherical polar coordinates $(r, \theta, \phi)$ as ${ }^{15}$

$$
\begin{aligned}
E_{r}(r, \theta, \phi)= & -l(l+1) P_{l}^{m}(\cos \theta) \frac{j_{l}\left(k_{l p} r\right)}{k_{l p} r} \\
& \times \exp \left(i m \phi-i \omega_{l p} t\right), \\
E_{\theta}(r, \theta, \phi)= & -\frac{\partial P_{l}^{m}(\cos \theta)}{\partial \theta} \frac{\left[k_{l p} r j_{l}\left(k_{l p} r\right)\right]^{\prime}}{k_{l p} r} \\
& \times \exp \left(i m \phi-i \omega_{l p} t\right), \\
E_{\phi}(r, \theta, \phi)= & -\frac{i m}{\sin \theta} P_{l}^{m}(\cos \theta) \frac{\left[k_{l p} r j_{l}\left(k_{l p} r\right)\right]^{\prime}}{k_{l p} r} \\
& \times \exp \left(i m \phi-i \omega_{l p} t\right),
\end{aligned}
$$

where $j_{l}$ is a spherical Bessel function and $P_{t}^{m}$ is an associated Legendre function. Outside the sphere the field distribution is obtained by the replacements $j_{l} \rightarrow h_{l}$ and $k_{l p} \rightarrow k_{l p} / n_{s}=\omega_{l p} / c$, where $h_{l}$ is an outgoing spherical Hankel function and $n_{s}$ is the index of refraction of the sphere. For each choice of $l$ the electromagnetic boundary conditions determine a characteristic equation whose roots give a discrete set of resonant wave numbers $k_{l p}$, with $l \approx k_{l 1} R$ for spheres of sufficiently large radius $R$. Note that the index $p$ corresponds to the number of maxima that $j_{l}\left(k_{l p} r\right)$ has in the interior region $r<R$.

The results of a numerical calculation for the cesium $6 S_{1 / 2} \leftrightarrow 6 P_{1 / 2}, 7 P_{3 / 2}$ manifold $\left(\lambda_{1}=894 \mathrm{~nm}\right.$, $\left.\lambda_{2}=456 \mathrm{~nm}\right)$ interacting with the $\operatorname{TM}(l=492$, $m=488, p=1)$ and $\operatorname{TM}(l=m=971, p=1)$ modes (corresponding to $\omega_{a}$ and $\omega_{b}$, respectively) in a quartz microsphere of radius $R=50.0404 \mu \mathrm{m}$ are shown in Fig. 2. To approximate the effects of cavity quantum electrodynamic level shifts, ${ }^{13}$ we have included a Van der Waals potential $U_{w}(\mathbf{r})=-\alpha /(r-R)^{3}$, with $\alpha \approx 30 \mathrm{~Hz} \mu \mathrm{m}^{3}$ for the cesium ground state, as given in Ref. 16. The well depth for the total potential $U(\mathbf{r}) \equiv U_{d}(\mathbf{r})+U_{w}(\mathbf{r})$ is $\sim 1 \mu \mathrm{K}$, and the well size is roughly $0.8 \mu \mathrm{m}$ in the $z$ direction and $0.13 \mu \mathrm{m}$ in the $\rho$ direction (FWHM). The centrifugal barrier associated with an atomic orbital kinetic energy $E_{\text {orb }}=40 \mu \mathrm{K}$ would flatten the well by $\sim 10 \%$. The saturation parameters $s_{1,2}(\mathbf{r})=1 / 2 \Omega_{1,2}(\mathbf{r}) /\left(\delta^{2}+\gamma_{1,2}^{2} / 4+\Omega_{1,2}^{2} / 2\right)$ are $<5 \times 10^{-7}$ within the well region, so the mean time for an atom to be heated out of the trap ${ }^{17}$ is quite long, roughly $4.5 \mathrm{~s}$. At $E_{\text {orb }}=10 \mu \mathrm{K}$ this time permits $\sim 450$ full orbits.

The stable orbits of the potential shown in Fig. 2 lie well within the region in which atoms can be strongly coupled to near-resonant optical modes. A quantitative measure of the strength of the atom-field interaction is given by the spatially varying coupling

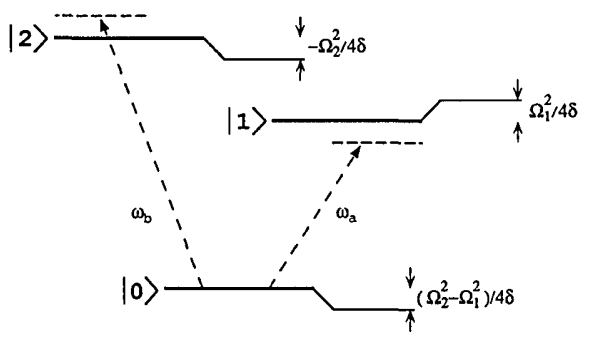

Fig. 1. Level diagram for an atom interacting with WGM's of frequencies $\omega_{a}$ and $\omega_{b}$. The first-order light shifts are indicated.

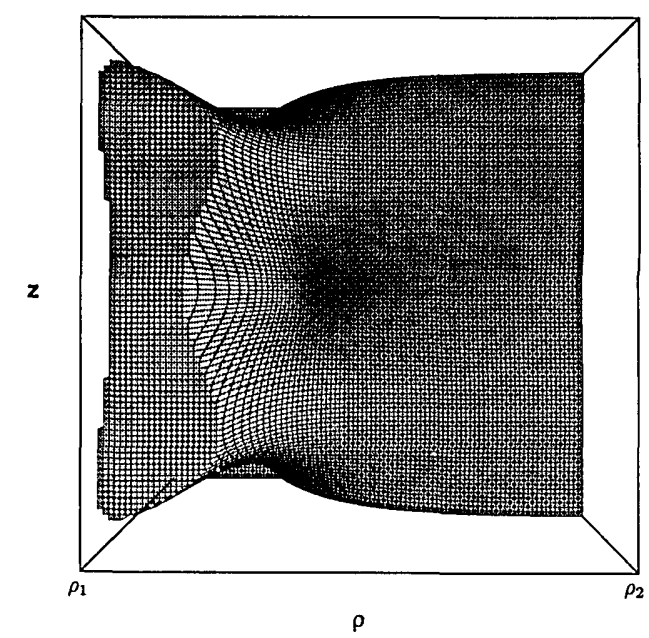

Fig. 2. Two-dimensional potential for atomic motion in the $(\rho, z)$ plane around a sphere of radius $R$, where $\rho$ is the radial and $z$ is the axial coordinate. The $\rho$ axis ranges from $\rho_{1}=R+0.0006 \mu \mathrm{m}$ to $\rho_{2}=R+0.5006 \mu \mathrm{m}$, the $z$ axis from -2 to $2 \mu \mathrm{m}$ (centered at zero), and the height of the potential from -1 to $+1 \mu \mathrm{K}$. The detunings of the WGM's used are $\delta_{1} / 2 \pi=-2.2 \times 10^{12} \mathrm{~Hz}$, $\delta_{2} / 2 \pi=2.3 \times 10^{12} \mathrm{~Hz}$. Note that the mode structure of the sphere does not permit $\left|\delta_{1}\right|=\left|\delta_{2}\right|$ exactly. Maximum Rabi frequencies in the well region of the external field are $\Omega_{1}^{*} / 2 \pi=3.45 \times 10^{8} \mathrm{~Hz}, \Omega_{2}^{*} / 2 \pi=3.58 \times 10^{8} \mathrm{~Hz}$. 


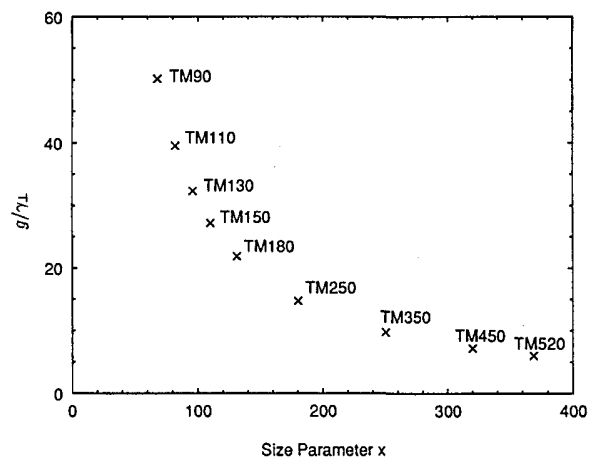

Fig. 3. Coupling coefficient $g / \gamma_{\perp}$ at $\mathbf{r}=(R, \theta / 2)$ versus size parameter $x=2 \pi R / \lambda$ for the cesium $D 2$ transition at $\lambda=852.36 \mathrm{~nm}\left(m_{F}=F=4 \rightarrow m_{F}^{\prime}=F^{\prime}=5\right)$ interacting with WGM's in microspheres of various size.

coefficient $g(\mathbf{r})$. Let $|\psi(\mathbf{r})| \propto|\mathbf{E}(\mathbf{r})|$ be the mode function of the WGM, normalized to have a maximum value equal to unity. Then $g(\mathbf{r})=\gamma_{\perp}|\psi(\mathbf{r})| \sqrt{V_{0} / V_{m}}$, where $V_{m}$ is the electromagnetic mode volume of the WGM and $V_{0}=3 c \lambda^{2} / 4 \pi \gamma_{\perp}$ is the effective volume of the atom for purely radiative interactions. Here $\gamma_{\perp}$ is the transverse decay rate of the transition of interest and $\mathbf{r}$ is the atom's position outside the microsphere. We take $V_{m}$ to be the integral of $|\epsilon(r) \psi(\mathbf{r})|^{2}$ over a spherical region of radius $R_{c}$ that includes both the interior of the sphere and the evanescent zone just outside the surface, where $\epsilon(r)$ is equal to the dielectric constant of quartz for $r<R$ and to 1.0 for $r>R$. The result of such a calculation is not sensitive to the exact value of the cutoff radius $R_{c}$ for $R_{\mathrm{c}}-R \gg \lambda / 2 \pi$.

The ratio $g(\mathbf{r}) / \gamma_{\perp}$ depends on the atom's position only through $|\psi(\mathbf{r})|$. We therefore present calculations of cavity quantum electrodynamic couplings for the case $g(r=R, \theta=\pi / 2) \equiv g$ only; coupling strengths for atoms located at arbitrary radii may be scaled by use of Eq. (1). Figure 3 shows $g / \gamma_{\perp}$ versus resonant size parameter $x=2 \pi R / \lambda$ for $\operatorname{TM}(l=m, p=1)$ WGM's in spheres of various sizes interacting with a particular hyperfine component of the cesium $D 2$ transition $\left(\lambda=852.36 \mathrm{~nm}, \gamma_{\perp} / 2 \pi=\right.$ $2.5 \mathrm{MHz}$ ). Note that $g / \gamma_{\perp}$ varies roughly as $R^{-2}$ over the displayed range of $x$. All modes shown have intrinsic $Q$ 's $>10^{12}$, so that the cavity-damping rates $\kappa$ for such modes should certainly be limited by bulk absorption and surface scattering in the microsphere. ${ }^{1}$

In a sphere of $50-\mu \mathrm{m}$ radius the relevant mode for the cesium $D 2\left(F=4 \rightarrow F^{\prime}=5\right)$ transition is $\operatorname{TM}(l=m=520, p=1)$. At the equilibrium radius $a=50.2 \mu \mathrm{m}$ for atoms trapped in the potential of Fig. 2 the scale factor $|\psi(a)| /|\psi(R)|$ determined from Eqs. (1) is 0.31 , which yields an expected coupling strength of $g(a) / \gamma_{\perp}=1.9$. For $Q \approx 2 \times 10^{9}$, as in Refs. 1 and $2, g(a) / \kappa \approx 54$. However, the data of Fig. 3 show that $g / \gamma_{\perp} \approx 50$ would be possible if one could bring cesium atoms very close to the surface of a sphere with $R \approx 9 \mu \mathrm{m}$. If $Q \approx 10^{11}$ could be achieved as well (as projected in Ref. 5), one would have $g / \kappa \approx 7.1 \times 10^{4}$. For comparison we note that, to our knowledge, the highest reported values achieved with cesium atoms in a Fabry-Perot cavity ${ }^{9}$ are $g / \gamma_{\perp}=2.9$ and $g / \kappa=12$. Note that, although our discussion has been limited to cesium atoms, yet higher values of $g / \gamma_{\perp}$ may be expected for transitions in other atoms (e.g., the $2 S \rightarrow 2 P$ transition in $\mathrm{He}^{*}$, for which $\mathrm{g} / \gamma_{\perp}=78$ with a sphere of $R \approx 11.7 \mu \mathrm{m}$ ), and improved trap parameters might be achievable as well.

Our calculations thus strongly encourage the pursuit of experimental cavity quantum electrodynamics with atoms strongly coupled to the external fields of WGM's in dielectric microspheres. The atom-gallery system described here promises not only large $g / \gamma_{\perp}$ and $g / \kappa$, but also the possibility of observing interesting mechanical effects of strong coupling. In addition, recent progress in laser cooling and atom interferometry suggests that the goal of confining atoms with $\Delta x \approx 2 \pi a$ to study de Broglie resonance phenomena may be achievable.

This study is supported by the National Science Foundation and the U.S. Office of Naval Research. H. Mabuchi is supported by an NDSEG fellowship. The authors gratefully acknowledge their ongoing collaboration with V. B. Braginsky's group at Moscow State University.

\section{References}

1. V. B. Braginsky, M. L. Gorodetsky, and V. S. Ilchenko, Phys. Lett A 137, 393 (1989); "Optical whisperinggallery microresonators," Proc. Soc. Photo-Opt. Instrum. Eng. (to be published).

2. L. Collot, V. Lefèvre-Seguin, M. Brune, J. M. Raimond, and S. Haroche, Europhys. Lett. 23, 327 (1993).

3. G. S. Agarwal and S. Duttagupta, Opt. Commun. 93, 173 (1992).

4. H. J. Kimble, H. Mabuchi, A. B. Matsko, and S. P. Vyatchanin, unpublished data.

5. P. W. Barber and R. K. Chang, eds., Optical Effects Associated With Small Particles (World Scientific, Singapore, 1988).

6. See session QTuC in Quantum Electronics and Laser Science, Vol. 12 of 1993 OSA Technical Digest Series (Optical Society of America, Washington, D.C., 1993), pp. 45-49.

7. A. Ashkin, Phys. Rev. Lett. 24, 156 (1970).

8. Y. B. Ovchinnikov, S. V. Shulga, and V. I. Balykin, J. Phys. B 24, 3173 (1991).

9. H. J. Kimble, in Cavity Quantum Electrodynamics, Advances in Atomic, Molecular, and Optical Physics, Supplement 2, P. R. Berman, ed. (Academic, San Diego, Calif., 1994).

10. V. I. Balykin and V. S. Letokhov, Appl. Phys. B 48, 517 (1989).

11. H. Wallis, J. Dalibard, and C. Cohen-Tannoudji, Appl. Phys. B 54, 407 (1992).

12. C. Cohen-Tannoudji and W. D. Phillips, Phys. Today 43(10), 33 (1990); S. Chu, Science 253, 861 (1991).

13. S. Haroche, in Fundamental Systems in Quantum Optics, Les Houches Summer School Session LIII, J. Dalibard, J. M. Raimond, and J. Zinn-Justin, eds. (Elsevier, Amsterdam, 1992), p. 771.

14. C. Cohen-Tannoudji, J. Dupont-Roc, and G. Grynberg, Atom-Photon Interactions (Wiley, New York, 1992).

15. J. A. Stratton, Electromagnetic Theory (McGraw-Hill, New York, 1941), p. 554.

16. M. Chevrollier, M. Fichet, M. Oria, G. Rahmat, D. Bloch, and M. Ducloy, J. Phys. II (Paris) 2, 631 (1992). 